\title{
Ueber eine Klasse auf einander abwickelbarer Flächen.
}

(Vòn' Herrn J. Weingarten.)

In der Theơrie der krummen Flăchen ist, wie es scheint, den Flächen der Krummungsmittelpunkte nur eine geringe Aufmerksamkeit zu Theil geworden. Die Bemerkwng jedoch, dass die abwickelbare Fläche, welche durch die längs einer Krümmungslinie einer gegebenen Fläche errichteten Normalen gebildet, wird; die entsprechende 'Fläche der Krümmungsmittelpunkte in einer geodätischen Linie ; schneidet, enthält, wenn wir nicht irren, die wesentlichste Eigenschaft dieser:Flächen. Ihre weitere Verfolgung, mit Rücksicht auf einige von Gauss in den: "Disquisitiones generales circa superficies ourvas" gegebene Theoreme, führt zu dem Satze, dass die Flächen der Krümmungsmittelpunkte derjenigen Flächen, bei denen in jedem Punkte der eine Hauptkrümmungsradius allein durch den anderen bestimmt ist, abgeschlossene Klassen auf einander abwickelbarer Flächen bilden. Dieser Satz, der mit der Theorie der auf Rotationsflächen abwickelbaren Flächen im innigsten Zusammenhange steht, ist es, welcher den Gegenstand der folgenden Mittheilung bildet.

Ist der Lauf einer krummen Oberfläche in der Art gegeben, dass die Coordinaten $x, y, z$ eines Punktes derselben bestimmt sind durch die Werthe zweier unabhängigen Variablen $p$ und $q$, und bezeichnen $X, Y, Z$ die Cosinus der Richtung der Normalen im Punkte $(x, y, z)$, so genügen diese Grössen den Gleichungen:

$$
\begin{aligned}
& X \frac{\partial x}{\partial p}+Y \frac{\partial y}{\partial p}+Z \frac{\partial z}{\partial p}=0 \\
& X \frac{\partial x}{\partial q}+Y \frac{\partial y}{\partial q}+Z \frac{\partial z}{\partial q}=0 \\
& X^{2}+Y^{2}+Z^{2}=1 .
\end{aligned}
$$

Es ist vortheilhaft dieselben in die folgende Form zu bringen:

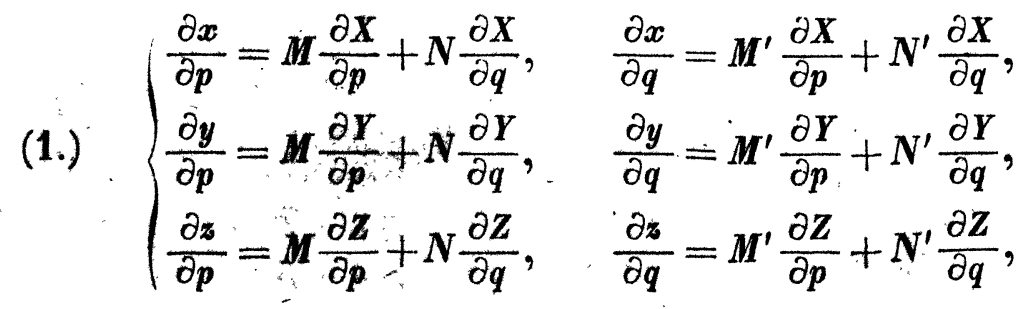


von welchen Gleichungen nach passender Bestimmung der Grossen $M, M^{\prime}, N, N^{\prime}$ aus vier dazu geeigneten die zwei übrigen eine Folge der fruheren sind $)$

Sind $(x, y, z)$ und $(x+d x, y+d y, z+d z)$ zwei unendlich nahe Punkte der krummen Fläche, welche derselben Krümmungslinie angehören, und messen $d X, d Y, d Z$ die Unterschiede der Cosinus der Richtung der Normalen fur beide Punkte, so bestehen bekanntlich die Gleichungen:

$$
\text { (2.) } d x=\varrho d X, d y=\varrho d Y, d z=\varrho d Z,
$$

in denen $\varrho$ den Hauptkrümmungsradius bezeichnet, welcher dem durch $(x, y, z)$ und $(x+d x, y+d y, z+d z)$ geführten Normalschnitte angehört; Gleichungen, die die Differentialgleichung der Krümmungslinien vertreten.

Eine Vergleichung dieser Gleichungen mit (1.) zeigt, dass die Differentialgleichung der Krümmungslinien in den Variablen $p$ und $q$ erhalten wird durch Elimination von $\varrho$ aus den Gleichungen

$$
\begin{aligned}
& M d p+M^{\prime} d q=\varrho d p, \\
& N d p+N^{\prime} d q=\rho d q,
\end{aligned}
$$

und dass ferner $\varrho$ bestimmt wird durch die quadratische Gleichung:

$$
\left|\begin{array}{cc}
M-\varrho & M^{\prime} \\
N & N^{\prime}-\varrho
\end{array}\right|=0 .
$$

Bezeichnet man die Hauptkrümmungsradien; welche dem durch die Werthe von $p$ und $q$ bestimmten Punkte der betrachteten Oberfläche angehören, durch $\rho$ und $\varrho^{\prime}$, so wird:

$$
\text { (3.) } \quad\left\{\begin{aligned}
\rho+\varrho^{\prime} & =M+N^{\prime}, \\
\rho \varphi^{\prime} & =M N^{\prime}-M^{\prime} N .
\end{aligned}\right.
$$

Aus diesen Beziehungen geht zugleich hervor, dass die Differentialquotienten der Coordinaten einer auf eine Ebene abwickelbaren Fläche nicht in die Form der Gleichungen (1.) gesetzt werden können, in welcher die Grössen $M$ und $N$ im Allgemeìnen endlich vorausgesetzt sind.

*) Die wirkliche Darstellung der Grössen $M, M^{\prime}, N, N^{\prime}$ ist für das Folgende entbehrlich. Eine leichte Rechnung ergiebt, wenn man sich der von Gauss eingeführten Bezeichnungen bedient:

$$
\begin{aligned}
& M=\frac{D^{\prime \prime} E-D^{\prime} F}{D D^{\prime \prime}-D^{\prime} D^{\prime}} \sqrt{E G-F F}, \quad N=\frac{-D E+D F}{D D^{\prime}-D^{\prime} D^{\prime}} \sqrt{E G-F F} \\
& M !=\frac{-D^{\prime} G+D^{\prime \prime} F}{D D^{\prime \prime}-D^{\prime} D^{\prime}} \sqrt{E G-F F}, \quad N^{\prime}=\frac{D G-D^{\prime} F}{D D^{\prime \prime}-D^{\prime} D^{\prime}} \sqrt{E G-F F}
\end{aligned}
$$


Die Variablen $p$, und $q$ bestimmen auf der vorgelegten Fläche zwei Systeme von Curven, in der Art, dass für das eine nur $p$, für das andere nur qeränderlich ist. Wir wählen für das Folgende diese Variablen so, dass dem ersten Systema eine Schaar von Krümmungslinien entspreche, dem weiten aber die Sehaar derjenigen Curven, für welche der Hauptkrümmungsradius, der dem gewählten Systeme von Krümmungslinien angehört, unverändert bleibt. Diesen Forderungen geschieht Genüge, wenn man die Variable $q$ aus der Bedingung bestimmt, dass $q=$ Const. die Gleichungen einer Krümmungslinienschaar darstellt, und wenn man für $p$ den dieser Schaar zugehörigen Krümmungsradius wählt. In Beziehung auf die Variable $q$ bleibe es vorbehalten, eine beliebige Function derselben als neue Variable einzuführen, wodurch die gestellten Bedingungen in Nichts verletzt werden.

Die Einführung der eben definirten Variablen $p, q$ als unabhängiger Variablen ist im Allgemeinen für jede Fläche auf zwei Weisen möglich; eine Ausnahme hiervon bilden offenbar die Ebene und die Kugel, für welche sie gar nicht, und diejenigen Flächen, deren einer Hauptkrümmungsradius längs der correspondirenden Krümmungslinie unverändert bleibt, wie z. B. die developpabelen und die Canalflächen, für welche diese Einführung nur auf eine Weise möglich ist.

Sind $\xi, \eta, \zeta$ die Coordinaten des dem Krümmungsradius $\varrho=p$ entsprechenden Krümmungsmittelpunktes, so ist:

$$
\text { (4.) } \quad x-\xi=p X, \quad y-\eta=p Y, \quad z-\zeta=p Z \text {. }
$$

In Beziehung auf den Punkt $\left(x+\frac{\partial x}{\partial p} d p, y+\frac{\partial y}{\partial p} d p, z+\frac{\partial z}{\partial p} d p\right)$ werden diese Gleichungen :

$$
\left\{\begin{array}{l}
\left(\frac{\partial x}{\partial p}-\frac{\partial \xi}{\partial p}\right) d p=p \frac{\partial X}{\partial p} d p+X d p \\
\left(\frac{\partial y}{\partial p}-\frac{\partial \eta}{\partial p}\right) d p=p \frac{\partial Y}{\partial p} d p+Y d p \\
\left(\frac{\partial z}{\partial p}-\frac{\partial \zeta}{\partial p}\right) d p=p \frac{\partial Z}{\partial p} d p+Z d p
\end{array}\right.
$$

Einer alleinigen Veränderung von $p$ entspricht, vermöge der Definition der Variablen $p, q$, ein Fortrücken vom Punkte $(x, y, z)$ zum Punkte $(x+d x$, $y+d y, z+d z)$ der Krümmungslinie $q=$ Const.

In Folge dessen ist nach den Gleichungen (2.)

$$
\frac{\partial x}{\partial p} d p=p \frac{\partial X}{\partial p} d p, \quad \frac{\partial y}{\partial p} d p=p \frac{\partial Y}{\partial p} d p, \quad \frac{\partial z}{\partial p} d p=p \frac{\partial Z}{\partial p} d p,
$$


und die Gleichungen (5.) ergeben:

$$
\text { (6.) } \begin{cases}\frac{\partial x}{\partial p}=p \frac{\partial X}{\partial p}, & X=-\frac{\partial \xi}{\partial p} \\ \frac{\partial y}{\partial p}=p \frac{\partial \boldsymbol{Y}}{\partial p}, & \boldsymbol{Y}=-\frac{\partial \eta}{\partial p} \\ \frac{\partial z}{\partial p}=p \frac{\partial Z}{\partial p}, & \boldsymbol{Z}=-\frac{\partial \zeta}{\partial p}\end{cases}
$$

Hiernach bestimmen sich die Coordinaten $x, y, z$ durch die Coordinaten des entsprechenden Krümmungsmittelpunktes folgendermassen:

$$
\text { (7.) } x=\xi-p \frac{\partial \xi}{\partial p}, \quad y=\eta-p \frac{\partial \eta}{\partial p}, \quad z=\zeta-p \frac{\partial \zeta}{\partial p} \text {. }
$$

Die Substitution dieser Werthe von $x, y, z, X, Y, Z$ in die Gleichung:

$$
\boldsymbol{X} \frac{\partial \boldsymbol{x}}{\partial \boldsymbol{q}}+\boldsymbol{Y} \frac{\partial y}{\partial q}+\boldsymbol{Z} \frac{\partial z}{\partial q}=\mathbf{0}
$$

führt auf die folgende:

$$
\frac{\boldsymbol{p}}{2} \frac{\partial\left[\left(\frac{\partial \xi}{\partial p}\right)^{2}+\left(\frac{\partial \eta}{\partial p}\right)^{2}+\left(\frac{\partial \zeta}{\partial p}\right)^{2}\right]}{\partial \boldsymbol{q}}-\left(\frac{\partial \xi}{\partial \boldsymbol{p}} \frac{\partial \xi}{\partial \boldsymbol{q}}+\frac{\partial \eta}{\partial \boldsymbol{p}} \frac{\partial \eta}{\partial \boldsymbol{q}}+\frac{\partial \zeta}{\partial \boldsymbol{p}} \frac{\partial \zeta}{\partial \boldsymbol{q}}\right)=\frac{\boldsymbol{p}}{2} \frac{\partial \boldsymbol{E}}{\partial \boldsymbol{q}}-\boldsymbol{F}=\mathbf{0},
$$

welche sich wegen:

in

$$
E=X^{2}+Y^{2}+Z^{2}=1
$$

verwandelt.

$$
\text { (8.) } \quad \mathbf{0}=\frac{\partial \xi}{\partial \boldsymbol{p}} \frac{\partial \xi}{\partial \boldsymbol{q}}+\frac{\partial \eta}{\partial \boldsymbol{p}} \frac{\partial \boldsymbol{\eta}}{\partial \boldsymbol{q}}+\frac{\partial \zeta}{\partial \boldsymbol{p}} \frac{\partial \zeta}{\partial \boldsymbol{q}}=\boldsymbol{F}
$$

Aus derselben folgt, dass auf der Fläche der Krümmungsmittelpunkte den Variablen $p$ und $q$ zwei Systeme von Curven entsprechen, welche sich in jedem Punkte unter rechten Winkeln schneiden. Dasjenige dieser Systeme, für welches allein $p$ veränderlich ist, ist, wie bekannt, ein System geodätischer Linien.

Vergleicht man die erste Reihe der Gleichungen (6.) mit den Gleichungen (1.), so bemerkt man, dass für die gewählten Variablen

$$
M=p, \quad N=\mathbf{0}
$$

wird. Die in (3.) gegebenen Bestimmungen der Hauptkrümmungsradien zeigen alsdann, dass $N^{\prime}$ identisch ist mit dem zweiten Hauptkrümmungshalbmesser $\varphi^{\prime}$, welcher in der Folge der Analogie wegen durch $p^{\prime}$ bezeichnet ist.

Mit Berücksichtigung dieser Bemerkungen und nach Substitution der Werthe von $x, y, z, X, Y, Z$ in $\xi, \eta, \zeta$, stellt sich die zweite Reihe der 
Gleichungen (1.) in folgender Form dar:

$$
\begin{aligned}
& \frac{\partial \xi}{\partial q}-p \frac{\partial^{2} \xi}{\partial p \partial q}=-M^{\prime} \frac{\partial^{2} \xi}{\partial p^{2}}-p^{\prime} \frac{\partial^{2} \xi}{\partial p \partial q}, \\
& \frac{\partial \eta}{\partial q}-p \frac{\partial^{2} \eta}{\partial p \partial q}=-M^{\prime} \frac{\partial^{2} \eta}{\partial p^{2}}-p^{\prime} \frac{\partial^{2} \eta}{\partial p \partial q} \\
& \frac{\partial \zeta}{\partial q}-p \frac{\partial^{2} \zeta}{\partial p \partial q}=-M^{\prime} \frac{\partial^{2} \zeta}{\partial p^{2}}-p^{\prime} \frac{\partial^{2} \zeta}{\partial p \partial q}
\end{aligned}
$$

welche Gleichungen, nachdem man sie der Reihe nach mit $\frac{\partial \xi}{\partial q}, \frac{\partial \eta}{\partial q}, \frac{\partial \zeta}{\partial q}$ multiplicirt und addirt hat, die Beziehung:

$$
\left(\frac{\partial \xi}{\partial \boldsymbol{q}}\right)^{2}+\left(\frac{\partial \boldsymbol{\eta}}{\partial \boldsymbol{q}}\right)^{\prime}+\left(\frac{\partial \zeta}{\partial \boldsymbol{q}}\right)^{2}=-\boldsymbol{M}^{\prime}\left(\frac{\partial \boldsymbol{F}}{\partial \boldsymbol{p}}-\frac{1}{2} \frac{\partial \boldsymbol{E}}{\partial \boldsymbol{q}}\right)+\frac{p-p^{\prime}}{2} \frac{\partial\left[\left(\frac{\partial \xi}{\partial \boldsymbol{q}}\right)^{2}+\left(\frac{\partial \eta}{\partial q}\right)^{2}+\left(\frac{\partial \zeta}{\partial q}\right)^{2}\right]}{\partial p}
$$

ergeben. Bezeichnet man durch $G$ die Summe:

$$
\left(\frac{\partial \xi}{\partial q}\right)^{2}+\left(\frac{\partial \eta}{\partial q}\right)^{2}+\left(\frac{\partial \zeta}{\partial q}\right)^{2}
$$

so wird, wenn man den, wegen $F=0$ und $\frac{\partial E}{\partial q}=0$, verschwindenden Theil unterdrückt:

und folglich:

$$
G=\frac{p-p^{\prime}}{2} \frac{\partial G}{\partial p}
$$

$$
\boldsymbol{G}=Q e^{2 \int \frac{d p}{p-p^{\prime}}}
$$

wo $Q$ eine Function von $q$ allein bezeichnet.

Die Functionen $\xi, \eta, \zeta$ genügen daher den Differentialgleichungen:

$$
\begin{aligned}
& \boldsymbol{E}=\left(\frac{\partial \xi}{\partial \boldsymbol{p}}\right)^{2}+\left(\frac{\partial \eta}{\partial \boldsymbol{p}}\right)^{2}+\left(\frac{\partial \zeta}{\partial \boldsymbol{p}}\right)^{2}=\mathbf{1}, \\
& \boldsymbol{F}=\frac{\partial \xi}{\partial \boldsymbol{p}} \frac{\partial \xi}{\partial \boldsymbol{q}}+\frac{\partial \eta}{\partial \boldsymbol{p}} \frac{\partial \eta}{\partial \boldsymbol{q}}+\frac{\partial \zeta}{\partial \boldsymbol{p}} \frac{\partial \zeta}{\partial \boldsymbol{q}}=\mathbf{0}, \\
& \boldsymbol{G}=\left(\frac{\partial \xi}{\partial \boldsymbol{q}}\right)^{2}+\left(\frac{\partial \eta}{\partial \boldsymbol{q}}\right)^{2}+\left(\frac{\partial \zeta}{\partial q}\right)^{2}=\boldsymbol{Q} e^{2 \int \frac{\partial p}{p-p^{\prime}}},
\end{aligned}
$$

in welchen Gleichungen man, unbeschadet der Allgemeinheit, $Q=1$ setzen kann, was mit der Einführung von

$$
\int \sqrt{ } Q d q
$$

als neuer Variablen aequivalent ist.

Dieso bemerkenswerthen Relationen führen unter der Voraussetzung, dass $\boldsymbol{p}^{\prime}$, d. i. der zweite Haquptkrümmungsradius der vorgelegten Fläche, nưr 
Weingarten, über eine Klasse auf einander abwickelburer Flächen.

von $\boldsymbol{p}$ abhänge, dass dieser also der Bedingung

$$
p^{\prime}=\lambda(\boldsymbol{p}),
$$

welcher ohne Weiteres eine partielle Differentialgleichung zweiter Ordnung zu substituiren ist, genüge, auf die oben ausgesprochenen mit der Theorie der abwickelbaren Flächen zusammenhängenden Folgerungen.

Unter dieser Voraussetzung erhält man:

$$
E=1, \quad F=0, \quad G=e^{2 \int \frac{\partial p}{p-\lambda(p)}}=\varphi(p) .
$$

Das Linienelement $d \sigma$ der Fläche der Krümmungsmittelpunkte, welche dem Radius $p$ entspricht, wird also:

$$
d \sigma^{2}=d \xi^{2}+d \eta^{2}+d \zeta^{2}=d p^{2}+\varphi(p) d q^{2}
$$

ein Ausdruck, welcher durch die Wahl der Variablen $p$ und $q$ dem Linienelemente der Fläche der Krümmungsmittelpunkte jeder krummen Oberfläche, welche der Differentialgleichung

$$
\boldsymbol{p}^{\prime}=\lambda(\boldsymbol{p})
$$

genügt, gegeben werden kann.

Es besteht daher folgendes Theorem:

Die Flächen der Krümmungsmittelpunkte aller Oberflächen, bei denen auf gleiche Weise in jedem Punkte der eine Hauptkrümmungsradius allein durch den anderen bestimmt ist, sind auf einander abwickelbar.

Es ist zu untersuchen, ob die durch das eben ausgesprochene Theorem gegebenen Flächen, deren Linienelement sich in die Form

$$
d p^{2}+\varphi(p) d q^{2}
$$

setzen lässt, die einzigen sind, denen diese Eigenschaft zukommt. Diese Form ist bekanntlich diejenige, die bei passender Bestimmung der Function $\varphi(p)$ dem Linienelemente einer jeden Umdrehungsfläche gegeben werden kann. Unsere Frage ist daher identisch mit der: ob eine jede auf eine gegebene Umdrehungsfläche aufwickelbare krumme Oberfläche in dem Systeme der Krümmungsmittelpunktsflächen einer durch die Gleichung $p^{\prime}=\lambda(p)$ characterisirten Flächenfamilie enthalten ist.

Es seien

$$
\begin{aligned}
u & =r \cos q \\
& =r \sin q \\
w & =F(r)
\end{aligned}
$$


die Gleichungen einer Rotationsfläche. Für das Quadrat des Linienelementes ergiebt sich:

Die Substitution

$$
d u^{2}+d v^{2}+d w^{2}=\left(1+F^{\prime}(r)^{2}\right) d r^{2}+r^{2} d q^{2}
$$

aus welcher folge:

$$
p=\int \sqrt{1+F^{\prime}(r)^{2}} d r
$$

gíebt demselben die verlangte Form:

$$
d u^{2}+d v^{2}+d w^{2}=d p^{2}+\varphi(p) d q^{2}
$$

Bezeichnen $\xi, \eta, \zeta$ die Coordinaten des dem Punkte $(u, v, w)$ bei der Abwickelung correspondirenden Punktes einer auf die Rotationsfläche abwickelbaren Fläche $S$. Drei bestimmten Werthen $u, v, w$ entsprechen drei zugehörige $\xi, \eta, \zeta$. Die ersten sind bestimmt durch gewählte Werthe von $p$ und $q$, in Folge dessen auch die letzten. Es sind daher $\xi, \eta, \zeta$ Functionen von $p$ und $q$, welche nach dem Begriffe der Abwickelbarkeit die Bedingung

$$
d u^{2}+d v^{2}+d w^{2}=d \xi^{2}+d \eta^{2}+d \zeta^{2}
$$

verificiren müssen. Sie erfüllen alsdann die partiellen Differentialgleichungen:

$$
\left\{\begin{aligned}
1 & =\left(\frac{\partial \xi}{\partial p}\right)^{2}+\left(\frac{\partial \eta}{\partial p}\right)^{2}+\left(\frac{\partial \zeta}{\partial p}\right)^{2} \\
0 & =\frac{\partial \xi}{\partial p} \frac{\partial \xi}{\partial q}+\frac{\partial \eta}{\partial p} \frac{\partial \eta}{\partial q}+\frac{\partial \zeta}{\partial p} \frac{\partial \zeta}{\partial q} \\
\varphi(p) & =\left(\frac{\partial \xi}{\partial q}\right)^{2}+\left(\frac{\partial \eta}{\partial q}\right)^{2}+\left(\frac{\partial \zeta}{\partial q}\right)^{2}
\end{aligned}\right.
$$

Man betrachte jetzt die Grössen

$$
\text { (10.) }\left\{\begin{array}{l}
x=\xi-p \frac{\partial \xi}{\partial p} \\
y=\eta-p \frac{\partial \eta}{\partial p} \\
z=\zeta-p \frac{\partial \zeta}{\partial p}
\end{array}\right.
$$

was : allgemein zu reden möglich ist, als die Coordinaten eines Punktes einer dritten Fläche $T$, und bezeichne der Kürze wegen durch $X, Y, Z$ die Differentialquotienten: $-\frac{\partial \xi}{\partial p},-\frac{\partial \eta}{\partial p},-\frac{\partial \zeta}{\partial p}$. Alsdann sind die Gleichungen

$$
\begin{aligned}
X d x+Y d y+Z d z & =0 \\
X^{2}+Y^{2}+Z^{2} & =1
\end{aligned}
$$


eine Folge der nach der Voraussetzung erfüllten Gleichungen (9.), und sie lehren, dass $X, Y, Z$ die Cosinus der Richtung der Normalen im Punkte $(x, y, z)$ der Fläche $T$ sind. Es ist leicht, die geometrische Bedeutung der Variablen $p, q$ in Beziehung auf diese Fläche zu ermitteln. Man hat nur die Werthe der Coordinaten $x, y, z$ und der Cosinus $X, Y, Z$ in die Gleichungen (1.) einzuführen, um zu bemerken, dass wegen der identisch erfüllten Gleichungen :

die Relationen

$$
\frac{\partial x}{\partial p}=p \frac{\partial \boldsymbol{X}}{\partial \boldsymbol{p}}, \quad \frac{\partial y}{\partial \boldsymbol{p}}=\boldsymbol{p} \frac{\partial \boldsymbol{Y}}{\partial \boldsymbol{p}}, \quad \frac{\partial \boldsymbol{z}}{\partial \boldsymbol{p}}=\boldsymbol{p} \frac{\partial \boldsymbol{Z}}{\partial \boldsymbol{p}},
$$

$$
M=p, \quad N=\mathbf{0}
$$

stattfinden. In Folge dessen erhält man zur Bestimmung der Hauptkrümmungsradien in einem Punkte der Fläche $\boldsymbol{T}$ die Gleichungen:

$$
\begin{aligned}
\varrho+\varrho^{\prime} & =p+N^{\prime}, \\
\varrho \varrho^{\prime} & =p N^{\prime} .
\end{aligned}
$$

Aus ihnen und dem Systeme der vorhergehenden ersieht man, dass die Grössen $q, p, N^{\prime}$ der Reihe nach mit dem Parameter der einen Schaar von Krümmungslinien der Fläche $T$, dem ihnen entsprechenden Hauptkrümmungsradius und dem anderen $\boldsymbol{p}^{\prime}$ identisch sind.

Eine schon durchgeführte Behandlung der zweiten Reihe der Gleichungen (1.) führt wieder auf die Gleichung

$$
\left(\frac{\partial \xi}{\partial q}\right)^{2}+\left(\frac{\partial \eta}{\partial q}\right)^{2}+\left(\frac{\partial \zeta}{\partial q}\right)^{2}=\frac{p-p^{\prime}}{2} \frac{\partial\left[\left(\frac{\partial \xi}{\partial q}\right)^{2}+\left(\frac{\partial \eta}{\partial q}\right)^{2}+\left(\frac{\partial \zeta}{\partial q}\right)^{2}\right]}{\partial p}
$$

welche sich vermöge der letzten der Gleichungen (9.) in

$$
\varphi(p)=\frac{p-p^{\prime}}{2} \varphi^{\prime}(p)
$$

verwandelt. Aus dieser ergiebt sich:

$$
\text { (11.) } \quad p^{\prime}=p-\sqrt{\varphi(p)} \frac{d p}{d \sqrt{\varphi(p)}}=\lambda(p) \text {. }
$$

Die Fläche $T$ gehört daher zu der Klasse derjenigen Flächen; bei denen in jedem Punkte der eine Hauptkrümmungsradius durch den anderen allein bestimmt ist. Sie hat die Fläche $S$, wie die Gleichungen (10.), wenn man ihnen die Form

$$
x-\xi=p X, \quad y-\eta=p Y, \quad z-\zeta=p Z
$$

giebt, lehren, zur Fläche der Krümmungsmittelpunkte. 
390 Wobightren, whe Klasse auf einander abwickelbarer Flächen.

Das Ergebniss unserer Untersuchung würde daher das Resultat sein, dass die Klasse derjenigen Flächen, deren Linienelement die Form

$$
d p^{2}+\varphi(p) d q^{2}
$$

gegeben werden kann, in der That congruent ist mit dem Systeme der dem Hauptkrümmungsradius $\varrho$ entsprechenden Schaalen der Flächen der Krümmungsmittelpunkte einer durch die partielle Differentialgleichung

$$
\varrho^{\prime}=\varrho-\sqrt{\varphi(\varrho)} \frac{d \varrho}{d \sqrt{\varphi(\varrho)}}=\lambda(\varrho)
$$

characterisirten Flächenfamilie, wenn nicht ein Umstand zu berücksichtigen wäre, der dieses Resultat, obgleich nicht wesentlich, beeinträchtigt. Die eben aus den Gleichungen (10.) gezogenen Folgerungen sind nämlich illusorisch in dem Falle, dass diese Gleichungen den Lauf einer Fläche nicht bestimmen. Alsdann aber sind die Grössen $x, y, z$ bestimmt durch die Werthe einer einzigen Veränderlichen $\tau$, die selbst Function beider Variablen $p, q$ oder einer von ihnen sein wird., Die in Folge der Gleichungen (9.) identisch erfüllten Gleichungen

$$
\begin{aligned}
& \frac{\partial x}{\partial p} \frac{\partial \xi}{\partial q}+\frac{\partial y}{\partial p} \frac{\partial \eta}{\partial q}+\frac{\partial z}{\partial p} \frac{\partial \zeta}{\partial q}=0 \\
& \frac{\partial x}{\partial \boldsymbol{q}} \frac{\partial \xi}{\partial \boldsymbol{q}}+\frac{\partial y}{\partial \boldsymbol{q}} \frac{\partial \eta}{\partial \boldsymbol{q}}+\frac{\partial z}{\partial \boldsymbol{q}} \frac{\partial \zeta}{\partial \boldsymbol{q}}=\varphi(p)-\frac{1}{2} p \varphi^{\prime}(p)
\end{aligned}
$$

werden unter dieser Voraussetzung:

$$
\left\{\begin{array}{l}
\left(\frac{\partial x}{\partial \boldsymbol{\tau}} \frac{\partial \xi}{\partial \boldsymbol{q}}+\frac{\partial y}{\partial \boldsymbol{\tau}} \frac{\partial \boldsymbol{\eta}}{\partial \boldsymbol{q}}+\frac{\partial z}{\partial \boldsymbol{\tau}} \frac{\partial \zeta}{\partial \boldsymbol{q}}\right) \frac{\partial \boldsymbol{\tau}}{\partial \boldsymbol{p}}=\mathbf{0} \\
\left(\frac{\partial \boldsymbol{x}}{\partial \boldsymbol{\tau}} \frac{\partial \xi}{\partial \boldsymbol{q}}+\frac{\partial y}{\partial \boldsymbol{\tau}} \frac{\partial \boldsymbol{\eta}}{\partial \boldsymbol{q}}+\frac{\partial \boldsymbol{z}}{\partial \boldsymbol{\tau}} \frac{\partial \zeta}{\partial \boldsymbol{q}}\right) \frac{\partial \boldsymbol{\tau}}{\partial \boldsymbol{q}}=\varphi(\boldsymbol{p})-\frac{1}{2} \boldsymbol{p} \varphi^{\prime}(\boldsymbol{p})
\end{array}\right.
$$

Das Bestehen dieser Gleichungen verlangt entweder

oder

$$
\frac{\partial \boldsymbol{x}}{\partial \boldsymbol{\tau}} \frac{\partial \boldsymbol{\xi}}{\partial \boldsymbol{q}}+\frac{\partial \boldsymbol{y}}{\partial \boldsymbol{\tau}} \frac{\partial \boldsymbol{\eta}}{\partial \boldsymbol{q}}+\frac{\partial \boldsymbol{z}}{\partial \boldsymbol{\tau}} \frac{\partial \zeta}{\partial \boldsymbol{q}}=\mathbf{0}
$$

$$
\frac{\partial \tau}{\partial p}=\mathbf{0}
$$

Ist die erste dieser Bedingungen erfüllt, so erfordert die zweite der Gleichungen (12.), dass:

und folglich:

$$
\text { (13.). } \varphi(p)=\frac{1}{2} p \varphi^{\prime}(p), \text { also: } \varphi(p)=c p^{2},
$$

$$
d \xi^{2}+d \eta^{2}+d \zeta^{2}=d p^{2}+c p^{2} d q^{2}
$$

eine Form des Linienelententes, die ntr einer auf eine Ebene abwickelbaren 
Fläche zukommt, welche Eigenschaft für die Fläche $\boldsymbol{S}$ nicht vorausgesetzt sein soll.

Die Erfüllung der anderen Bedingung $\frac{\partial \tau}{\partial p}=0$ reducirt die drei Grössen $x, y, z$ auf drei Functionen $V, V^{\prime}, V^{\prime \prime}$ von $q$ allein, wonach $\xi, \eta, \zeta$ von der Form

$$
\left\{\begin{array}{l}
\xi=p U+V, \\
\eta=p U^{\prime}+V^{\prime}, \\
\zeta=p U^{\prime \prime}+V^{\prime \prime}
\end{array}\right.
$$

sein müssen, in welchen Gleichung $U, U^{\prime}, U^{\prime \prime}$ ebenfalls nur durch $q$ bestimmte Grössen andeuten. Da in dem Linienelemente der durch diese Gleichungen dargestellten Flächen der Coefficient $\varphi(p)$ von $d q^{2}$ nur Function von $p$ sein soll, so tritt der zu behandelnde Fall nur ein, wenn

$$
\varphi(p)=\alpha p^{2}+2 \beta p+\gamma,
$$

wo $\alpha, \beta, \gamma$ Constante bezeichnen, oder ebenso allgemein durch eine Verschiebung des Anfangspunktes von $p$, wenn

$$
\text { (15.) } \varphi(p)=\alpha p^{2}+\gamma \text {. }
$$

Diese Form von $\varphi(p)$, in welcher die durch (13.) gegebene enthalten ist, ist daher die einzige, mit welcher das Eintreten des eben erörterten Umstandes verknüpft sein kann. Zur vollständigen Ermittelung der Flächen, deren Linienelement sich in die Form

$$
d \sigma^{2}=d p^{2}+\left(\alpha p^{2}+\gamma\right) d q^{2}
$$

bringen lässt, ist folglich die Kenntniss der durch die aus (11.) hervorgehende partielle Differentiaigleichung

$$
\rho \rho^{\prime}=-\frac{\gamma}{\alpha}
$$

definirten Flächen nicht hinreichend, sondern es ist noch den entsprechenden Krümmungsmittelpunktsflächen die Familie der aus geraden Erzengungslimien gebildeten Flächen (14.) mit der Bedingung, dass das Linienelement die verlangte Form annimmt, beizugesellen. Es hat keine Schwierigkeit, die sechs Functionen $U$ und $V$ der Gleichungen (14.) dieser Beđ̛̣ingung gemäss durch eine willkürlich bleibende zu bestimmen. Unter den durch sie dargestellten Flächen ist z. B. die Schraubenfläche .

$$
\xi=p \cos q, \quad \eta=p \sin q, \quad \zeta=w q
$$

enthalten, deren Linienelement gleich $d p^{2}+\left(p^{2}+a^{2}\right) d q^{2}$ wird, und die sich nicht 
wie die Umdrehungsfläche der Kettenlinie

$$
y=\frac{a}{2}\left(e^{\frac{x}{a}}+e^{-\frac{x}{a}}\right)
$$

welche dasselbe Linienelement liefert, als die Fläche der Krümmungsmittelpunkte einer Fläche von constanter negativer Krümmung $-\frac{1}{a^{2}}$ ansehen lässt.

Aus dem ehen Bewiesenen ergeben sich folgende auf die Theorie der auf Rotationsflächen abwickelbaren Flächen bezügliche Sätze, welche, nach Erledigung des auf die partielle Differentialgleichung $\rho \varrho^{\prime}=c$ bezüglichen Ausnahmefalles, ohne Rücksicht auf denselben ausgesprochen sind:

Das System der dem Hauptkrümmungsradius $\rho$ entsprechenden Schaalen der Flächen der Krümmungsmittelpunkte einer durch die partielle Differentialgleichung

$$
\rho^{\prime}=\lambda(\rho)
$$

characterisirten Flächenfamilie, constituirt eine abgeschlossene Klasse von auf einander abwickelbaren Flächen, als deren Repräsentant eine mit der Function $\lambda$ bestimmte Rotationsfläche angesehen werden kann.

Und umgekehrt:

Die Klasse der auf eine gegebene Rotationsfläche abwickelbaren Flächen ist congruent mit dem Systeme der dem Radius $\varrho$ entsprechenden Flächen der Krümmungsmittelpunkte einer durch die partielle Differentialgleichung

$$
\rho^{\prime}=\lambda(\varphi)
$$

charakterisirten Fiächenfamilie, in welcher die Function $\lambda$ der vorgelegten Rotationsfläche gemäss zu bestimmen ist.

Diese Theoreme, welche eine nothwendige und hinreichende Eigenschaft der auf Rotationsflächen auflegbaren Flächen kennen lehren, führen wenigstens in einem Falle zur endlichen Darstellung einer vollständigen Klasse von auf eine specielle Rotationsfläche abwickelbaren Flächen. Es ist dies der Fall, in welchem die zwischen $\varphi^{\prime \prime}$ und $\varphi^{\prime}$ stattindende Gleichung diejenige der Flächen kleinster Oberfläche

$$
\varphi+\varphi^{\prime}=0
$$

wird, deren Integration bekannt ist. Unter dieser Bedingung ist der gemeinschaftliche Ausdruck des Linienelementes aller Krümmungsmittelpunktsflächen dieser Flächenfamilie:

$$
d \sigma^{2}=d p^{2}+p d q^{2}
$$


Die Umdrehungsfläche, deren Linienelement diese Form annimmt, findêt ch durch eine leichte Rechnung als durch die Gleichungen

$$
\begin{aligned}
& \zeta=-\frac{r}{2} \sqrt{4 r^{2}-1}+\frac{1}{4} \log \left(2 r+\sqrt{4 r^{2}-1}\right) \\
& r=\sqrt{\xi^{2}+\eta^{2}}
\end{aligned}
$$

gegeben. In der That, setzt man:

$$
\begin{aligned}
\xi & =\gamma p \cos q \\
\eta & =\gamma p \sin q \\
\zeta & =-\frac{1}{2} \sqrt{p(4 p-1)}+\frac{1}{4} \log (2 \sqrt{ } / p+\sqrt{4 p-1)}
\end{aligned}
$$

so ergiebt sich:

$$
d \xi^{2}+d \eta^{2}+d \zeta^{2}=d p^{2}+p d q^{2}
$$

wie verlangt wurde. Die Meridiancurve dieser Umdrehungsfläche ist, wie man weiss, die Evolute der Kettenlinie:

$$
y=\frac{1}{8}\left(e^{4 x}+e^{-4 x}\right),
$$

ein Resultat, welches auch ohne Rechnung ersichtlich ist, wenn man sich daran erinnert, dass die Umdrehungsfläche einer jeden Kettenlinie (um die entsprechende Axe) eine Fläche ist, welche der partiellen Differentialgleichung

$$
\varphi+\varphi^{\prime}=0
$$

Genüge leistet, und folglich ist die Umdrehungsfläche ihrer Evolute die entsprechende Fläche der Krümmungsmittelpunkte.

Die Flächen der Krümmungsmittelpunkte der Flächen kleinster Oberfläche bilden daher die Klasse der auf die Rotationsfläche einer oder richtiger jeder Kettenlinienevolute abwickelbaren Flächen.

Was schliesslich die auf eine Ëbene abwickelbaren FIãchen betrifft, wolche sich unseren Betrachtungen wegen der Form, in welcher dieselben gefuhrt wurden, entzogen haben, so überzeugt man sich leicht und auf verschiedenen Wegen, dass die Flächen ihrer Krümmungsmittelpunkte, wenn solche existiren, wiederum auf eine Ebene abwickelbare Flächen sind.

Berlin, im Juni 1861. 